\title{
Ascending and Aortic Arch Thrombus: Cause of Myocardial Infarction and Lower Extremity Ischemia
}

\author{
Nneoma Olivia Okoronkwo ${ }^{\mathrm{a}, \mathrm{c}}$, Frank Wang ${ }^{\mathrm{a}}$, Gabriele Di Luozzo ${ }^{\mathrm{b}}$
}

\begin{abstract}
Ascending aortic thrombus is a rare finding that might lead to lifethreatening thromboembolic events. With no specific guidelines, controversy exists regarding management ranging from surgical resection, thrombolytic therapy, and thrombectomy to anticoagulation therapy. We report a particularly interesting case of a 61-year-old woman presenting with NSTEMI and lower extremity claudication from an embolic thrombus in the distal ascending aorta and proximal aortic arch treated medically with anticoagulation. Repeat imaging in 2 months revealed resolution of the thrombus.
\end{abstract}

Keywords: Ascending aorta; Aortic thrombus; Anticoagulation

\section{Introduction}

Ascending aortic thrombus is a rare finding and in most reported aortic thrombus cases [1] presents as cerebral or peripheral embolic complications. Treatment modalities of aortic thrombi vary [1], with few examples of conservative treatment [2]. We report a particularly interesting case of a patient presenting with NSTEMI and lower extremity claudication from an embolic thrombus in the distal ascending aorta and proximal aortic arch treated medically with anticoagulation.

\section{Case Report}

A 61-year-old woman with hypertension presented to the emergency room with 3-week history of left calf pain and numbness

Manuscript accepted for publication February 13, 2015

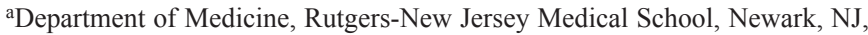
USA

bivision of Cardiac Surgery, Hackensack University Medical Center, Hackensack, NJ, USA

${ }^{\mathrm{c} C}$ Corresponding Author: Nneoma Olivia Okoronkwo, Department of Medicine, Rutgers-New Jersey Medical School, 150 Bergen Street, UH-I248, Newark, NJ 07101, USA. Email: okoronnn@njms.rutgers.edu

doi: http://dx.doi.org/10.14740/jmc2074w of left first toe that worsened with exertion and was relieved by rest. While in the emergency room, she developed mid-sternum chest tightness that lasted for $5 \mathrm{~min}$ and resolved spontaneously. Vital signs revealed a blood pressure of 191/109 $\mathrm{mm} \mathrm{Hg}$ and cardiopulmonary examination was unremarkable. Her left lower extremity was warm to touch with no palpable dorsalis pedis or posterior tibial pulses. Initial troponin I was $0.02 \mathrm{ng} / \mathrm{mL}$, increasing to $2.91 \mathrm{ng} / \mathrm{mL}$, and peaked at $3.15 \mathrm{ng} /$ $\mathrm{mL}$ (normal, $<0.05 \mathrm{ng} / \mathrm{mL}$ ). Electrocardiogram showed new $\mathrm{T}$-wave inversions in leads V3-V6 as well as flattened T waves in leads II and avF.

Left heart catherization showed $60 \%$ non-obstructive lesion of the distal left anterior descending artery. Angiogram of left extremity showed above-knee popliteal artery occlusion with reconstitution below the knee. TTE showed normal LV systolic function (ejection fraction of $60 \%$ ) with no evidence of intracardiac thrombus or thoracic aortic atheroma. Patient was medically managed with aspirin, heparin infusion, betablockers, statins and nitroglycerin.

CT angiogram of the chest done to evaluate source of peripheral emboli revealed an irregular linear filling defect noted in the posterior aspect of the ascending aorta consistent with a clot. Cardiac MRI showed two lesions in the distal ascending aorta and proximal arch, the largest measuring $7 \times 7 \mathrm{~mm}$ located approximately $7 \mathrm{~cm}$ from the aortic root (Fig. 1a). The second lesion was $2 \mathrm{~cm}$ distal on inferior wall of the aortic arch (Fig. 1b). Patient was managed with heparin then bridged to warfarin with an INR goal of 2.5 - 3.5. Repeat cardiac MRI done 2 months later showed resolution of previous linear filling defect suggesting resolution of clot (Fig. 1c, d). Laboratory tests for hypercoagulable states were sent; anti-thrombin III, protein C, protein S, homocysteine, factor VIII, ANA, and anti-cardiolipin antibodies returned within normal values.

\section{Discussion}

The ascending aorta is an uncommon site for thrombus formation due to high blood flow and sheer stress. The mechanism of thrombus formation in the ascending aorta remains unclear, but histological analysis of aortic intima in another publication has shown degenerative changes to aortic lining with loss of endothelium, fragmentation of underlying elastin, and fibrin deposits on the aortic surface [3]. 

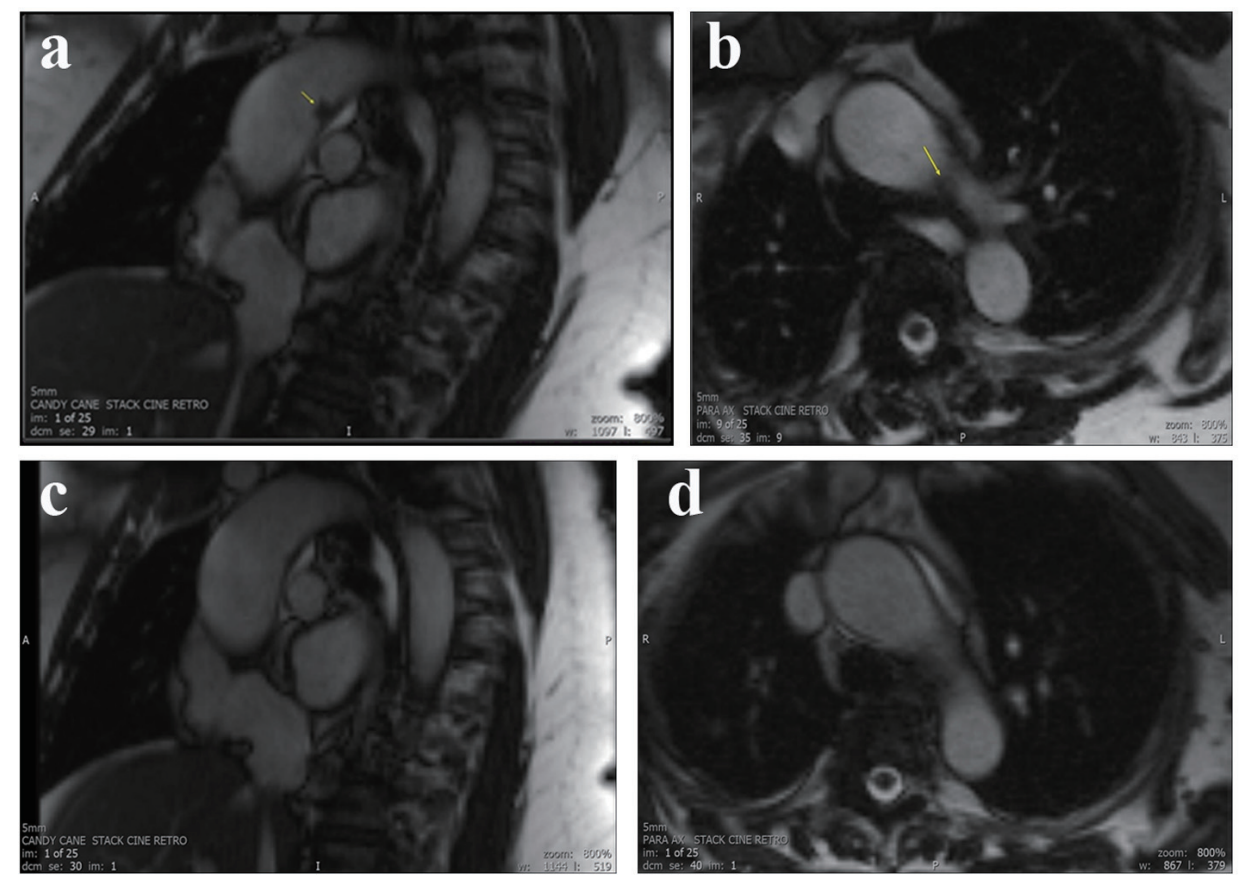

Figure 1. Diagnostic imaging: (a) magnetic resonance angiogram of ascending aortic thrombus; (b) magnetic resonance angiogram of proximal aortic arch thrombus; (c) follow-up magnetic resonance angiogram of ascending aorta; (d) follow-up magnetic resonance angiogram of proximal aortic arch.

Ascending aortic thrombus may lead to life-threatening thromboembolic events, warranting immediate management. With no clear guidelines, controversy exists regarding the management of ascending aortic thrombus. There are various methods employed in the management of aortic thrombus including anticoagulant therapy [2], thrombolytic therapy [4], thrombectomy [5], and surgical resection [3]. Currently no evidence suggests one method is preferable over the others, therefore treatment of choice remains problematic. There is a theoretical possibility that anticoagulation could cause fragmentation of thrombus with massive embolism and significant neurologic sequelae. During anticoagulation, size reduction of thrombus should be observed until the thrombus is completely resolved.

As of yet, we do not have a thorough understanding of the pathophysiology of the formation of an aortic thrombus especially in relatively young patients without any typical risk factors for cardiovascular disease. We performed an MRA of the chest for further delineation of the aortic wall and mobility of the aortic thrombus since this area of the ascending aorta and aortic arch is difficult to visualize on transthoracic and transesophageal echocardiogram. The MRA indicated that the base of the thrombus was wide and immobile; however, there were a few, very thin projectile strands that were quite mobile. A piece of these thin projections most likely embolized through the left main coronary artery during diastole leading to the myocardial infarction. The overall description and improved clinical scenario allow us to consider conservative management and successful resolution of the thrombus. Since the etiology was unclear, we plan on continuing anticoagulation therapy with imaging of the aorta every 6 months for 1 year.

In brief, this is an interesting presentation of a middleaged woman with ascending and aortic arch thrombi successfully treated conservatively with anticoagulation. Thrombi in such locations are rare, but should be considered as a possible source of systemic embolism. Further investigation is needed to understand risk factors and evaluate the various treatment modalities for this rare condition.

\section{Grant Support}

None.

\section{Conflict of Interest}

No disclosures.

\section{References}

1. Choukroun EM, Labrousse LM, Madonna FP, Deville C. Mobile thrombus of the thoracic aorta: diagnosis and treatment in 9 cases. Ann Vasc Surg. 2002;16(6):714-722.

2. Blackshear JL, Jahangir A, Oldenburg WA, Safford RE. Digital embolization from plaque-related thrombus in the thoracic aorta: identification with transesophageal echocardiography and resolution with warfarin therapy. Mayo Clin Proc. 1993;68(3):268-272.

3. Soo A, Marley D, McGrath-Soo L, Herron B, Parissis H. 
Ascending aorta thrombus: A diagnostic and treatment dilemma. Asian Cardiovasc Thorac Ann. 2013;22(6):731733.

4. Hausmann D, Gulba D, Bargheer K, Niedermeyer J, Comess KA, Daniel WG. Successful thrombolysis of an aortic-arch thrombus in a patient after mesenteric embolism. N Engl J Med. 1992;327(7):500-501.

5. Haida H, Inoue Y, Kawajiri H, Suzuki S. Rod-shaped giant floating thrombus in a normal ascending aorta. Eur J Cardiothorac Surg. 2015;47(3):582. 